Original article

Paediatrics Today 2015;11(2):144-153

DOI $10.5457 / \mathrm{p} 2005-114.120$

\title{
THE EFFECT OF OXYGEN THERAPY ON THE DEVELOPMENT OF RETINOPATHY OF PREMATURITY
}

\author{
Dragica JOJIĆ ${ }^{*}$, Jelica PREDOJEVIĆ-SAMARDŽIĆ ${ }^{1}$, Milka MAVIJA², Dimitrije NIKOLIĆ ${ }^{3}$, \\ Vesna MARINKOVIĆ 4
}

${ }^{1}$ Department of Pediatrics, University Hospital Clinical Centre, Banja Luka Bosnia and Herzegovina, ${ }^{2}$ Department of Ophthalmology, University Hospital Clinical Centre, Banja Luka, Bosnia and Herzegovina, ${ }^{3}$ University Children's Hospital, Belgrade, Serbia, ${ }^{4}$ Medical Faculty of Belgrade University, Belgrade, Serbia

\footnotetext{
${ }^{*}$ Corresponding author: dragajojic@gmail.com Tel.: + 38751342408

Fax.: + 38751342463
}

Received: July 17, 2015

Accepted: August 14, 2015

Key words: Retinopathy of prematurity - Oxygen therapy - Risk factors

- Premature infant.

\begin{abstract}
Objective - The aim of this study is to show the effect of oxygen therapy in premature babies on the development of severe ROP. Materials and methods - This retrospective research covered a period of two years, and included 108 premature babies, with birth weight $<1500 \mathrm{~g}$ and gestational age $<33$ weeks, at the Children's Ward of the University Hospital Clinical Centre, Banja Luka. The effects of the length of oxygen therapy and episodes of hypoxia and hyperoxia on the development of severe ROP were analysed. Results - ROP was present in $64(59.2 \%)$ newborns, of which 21 (19.4\%) had severe ROP. The incidence of severe ROP is statistically higher with lower birth weight, $<1150 \mathrm{~g}(\mathrm{p}<0.01)$, lower gestational age $<30$ weeks, longer oxygen therapy and when there were frequent episodes of hyperoxia and hypoxia. By multiple logistic regression analysis, a very strong connection was established between severe ROP and exposure to episodes of hyperoxia (OR 32.73; CI 4.14-255.37), episodes of hypoxia (OR 14.08; CI 3.81-51.94), and oxygen therapy longer than 10 days (OR 15.54; CI 1.99-120.79). For most risk factors tested, there was a strong connection between severe ROP and birth weight $<1250$ g, gestational age $<30$ weeks, respiratory distress syndrome and perinatal asphyxia. Conclusion - Long-term oxygen therapy, prematurity and low birth weight with hypoxia are important factors in the development of severe ROP. Constant education is necessary of staff regarding the potentially harmful effects of oxygen in order to prevent both hypoxia and hyperoxia.
\end{abstract}

\section{Introduction}

Retinopathy of prematurity (ROP) is an illness of the immature retina, which causes severe damage to sight in babies with low gestational age and low birth weight. Several risk factors are responsible for the development of retinopathy of prematurity, which prevent the normal development of vascularization of the retina. One of the most important is the effect of oxygen therapy in premature babies with low gestational age (1).

Oxygen was first used in newborns who did not breathe spontaneously by Chaussier in 1780 . The use of oxygen in neonatology has been routine since the 1950 's, but it was quickly suspected that there was a connection between the use of oxygen and the occurrence of retinopathy of prematurity (2). 
Oxygen was blamed as the main cause of ROP, and its use was reduced, and the level of oxygen provided in the air inhaled was set at $40 \%$ (3) This led to a significant reduction in the incidence of this illness, but there was an increase in neurological and respiratory complications, as well as an increase in mortality of premature babies. Once again from 1970 the number of children with ROP increased, due to the survival of children with very low birth weight, below $1000 \mathrm{~g}$, and at less than 28 weeks gestational age (2). Continuous monitoring was introduced of oxygen concentrations through the skin using a pulse oximeter from 1980 (4), and the more intensive monitoring began of oxygen concentrations in blood samples. After the introduction of controlled use of additional oxygen, it was shown that high oxygen levels were not the only significant risk factor for the occurrence and development of ROP. The incidence of the disease persists, indicating the effect of other factors on the genesis of ROP, which has resulted in many clinical studies (5). The pathogenesis of ROP has still not been completely explained. Two phases of retinopathy have been described, in which a key role is played by oxygen. In the first phase of retinopathy, with premature birth the normal development of blood vessels which began in utero is interrupted. The initial damage is caused by hyperoxia, with the creation of free formations of radicals, which cause damage to the development of new blood vessels, and interrupt the normal process of angiogenesis. In the second phase of ROP, with maturity the metabolic needs of the retina increase and hypoxia becomes more expressed. Hypoxia stimulates the increased creation of Vascular Endothelial Growth Factor (VEGF) and causes pathological neovascular retinas at about 32-34 weeks gestational age (6).

From 1980 to 1990 the treatment began of severe forms of ROP using cryotherapy, and later laser photocoagulation (7). Recently use began of intravitreal administration of the VEGF inhibitor, bevacizumab (8). Early complications of ROP can be halted or caused to regress by timely treatment (2).

The aim of this study is to show the effect of oxygen therapy in premature babies on the development of severe ROP.

\section{Materials and methods}

Over a two-year period, a retrospective study was undertaken at the Children's Ward of the University Hospital Clinical Centre, Banja Luka. We selected 108 premature babies who met the criteria for the ophthalmology screening program for ROP. For the study, babies were excluded who had major congenital malformations, chromosome anomalies and newborns who died before the ophthalmology examination. The subjects were divided into 3 groups: newborns without ROP; newborns with stage I and IIROP; newborns with severe ROP requiring surgical intervention.

Screening for ROP was undertaken in premature babies of gestational age $<33$ weeks and birth weight $<1500 \mathrm{~g}$, and in newborns with additional risks for development of ROP. The criteria of ophthalmology screening for retinopathy of prematurity were accepted according to those proposed by the American Academy of Pediatrics (9). At-risk premature babies were selected for screening by paediatrician-neonatologists. Maximum mydriasis was achieved using $0.5 \%$ cyclopentolate eye drops and immediately before the examination the anaesthetic benoxinate at $0.4 \%$. The examination was performed using an indirect binocular ophthalmoscope and $20 \mathrm{D}$ magnifying lens. For a better view of the peripheral parts of the retina an indentor was also used. During the screening the development was monitored of the blood vessels in the retina, and premature babies with severe cases of retinopathy of prematurity were separated out, where treatment prevented the further progress of the pathological process (2). The anatomical classification 
of the scope of localization, extent and stage of the pathological changes to the vascularization of the retina was according to the International Classification of Retinopathy of Prematurity, ICROP) (9). For each newborn with ROP, the age at which ROP was detected, the maximum stage of ROP and the treatment of ROP were defined.

All premature babies underwent continuous monitoring of percutaneous values of arterial haemoglobin oxygen saturation $\left(\mathrm{SaO}_{2}\right)$ using a pulse oximeter. For measurement of saturation a NellcorOxiMax N-560 pulse oximeter was used, with an OXIMax sensor, or a Patient Monitor YM6000 with an Oximax sensor. Additional oxygen was administered to maintain $\mathrm{SaO}_{2}<92 \%$ measured by the pulse oximeter. Oxygen is used in treatment in the form of a controlled increase in concentrations of inspired oxygen (using a diffusion atmospheric incubator or "hood"), non-invasive positive pressure ventilation using nasal CPAP or biphasic mode ventilation, with Infant Flow SiPAP, Viasys Healthcare apparatus, and conventional mechanical ventilation, using Dräger Babylog 8000 apparatus. Respiratory therapy includes: the duration of oxygen therapy and the type of respiratory support. The duration of use of oxygen is expressed in days, and for mechanical ventilation, whether it was used or not.

Gas analysis and acid base status were established from samples of capillary blood from the heel, by a ABL 800 FLEX machine. The values of gas analysis - haemoglobin oxygen saturation, partial pressure $\mathrm{O}_{2}$ and $\mathrm{CO}_{2}$ and $\mathrm{pH}$ were established 1-4 times a day, depending on the child's clinical status. Hyperoxia is defined as values of $\mathrm{pO}_{2}>12 \mathrm{kPa}$ and more, and hypoxia as values of $\mathrm{pO}_{2}<5.3 \mathrm{kPa}$. A significant number of hyperoxia episodes was defined as 10 or more episodes. The average oxygen fraction in the inspired air in the first two weeks of life was established in order to assess the severity of lung damage.
The degree of respiratory distress syndrome (RDS) was assessed on the basis of clinical and radiology findings of the lungs, classified according to Bomsell, from I-V. Newborns with severe RDS were given a surfactant. Perinatal asphyxiation was assessed on the basis of the clinical picture, the Apgar score and the acid base status. The Apgar was determined by the obstetrician in the labour ward in the case of vaginal delivery, and by the paediatrician-neonatologist in the case of Caesarean delivery. Perinatal asphyxia, respiratory distress syndrome and apnoea were considered to be present or absent. The demographic data analysed were: gestational age in weeks, birth weight in grammes and gender.

\section{Statistical analysis}

For the sake of data analysis, the statistical package SPSS for Windows 20.0 was used. Data were processed using descriptive statistical methods. Univariate comparison of risk factors between groups without ROP, newborns with ROP not requiring surgery and severe ROP was performed using the Student T-test and the Hi-square test with the level of significance at $\mathrm{p}<0.05$. Multivariate logistic regression was used to assess predictive factors for development of ROP. The odds ratio and a $95 \%$ confidence interval for each risk factor were calculated.

\section{Results}

Ophthalmology screening for ROP encompassed 108 premature babies, 60 males (55.5\%) and 48 (44.4\%) females. The first ophthalmology screening examination in all newborns was performed at between 31 and 37 gestational weeks. ROP was present in 64/108 (59.2\%), of which 21/108 (19.4\%) had severe ROP, and the results were normal in $44 / 108(40.7 \%)$.

The birth weight of newborns with severe ROP was $1154.3 \pm 224.9$ and gestational age 
$28.8 \pm 1.5$ weeks. Premature babies without ROP had birth weight $1772.7 \pm 597.3$ and gestational age $31.6 \pm 1.3$ weeks (Table 1 ).

The incidence of severe ROP was statistically significantly more frequent as the birth weight was lower, birth weight $<1250$ $\mathrm{g}(\mathrm{p}<0.01)$, lower gestational age $<30$ weeks, and if the premature babies suffered perinatal asphyxia and had frequent apnoea. The incidence of severe ROP is statistically significantly more frequent with more severe levels of respiratory distress due to Hyaline Membrane Disease $(\mathrm{p}<0.05)$ (Table 2$)$.

\section{Table 1 Anthropometric characteristics of preterm infants}

\begin{tabular}{lllll}
\hline \multirow{2}{*}{ Anthropometric characteristics } & \multicolumn{3}{l}{ Retinopathy of prematurity } \\
\cline { 2 - 4 } & \multicolumn{2}{l}{ Without } & I and II stage & Severe \\
\cline { 2 - 4 } & $\mathrm{n}=44$ & $\mathrm{n}=43$ & $\mathrm{n}=21$ \\
\hline \multirow{2}{*}{ Gestational age $\left(\right.$ weeks; $\left.\overline{\mathrm{X}}_{ \pm} \mathrm{SD}\right)$} & $31,6 \pm 1,3$ & $30,8 \pm 1,4$ & $28,8 \pm 1,5$ \\
\multicolumn{2}{l}{ Birth weight $\left(\mathrm{g} ; \overline{\mathrm{X}}_{ \pm} \mathrm{SD}\right)$} & $1772,7 \pm 253,9$ & $1469,6 \pm 282,2$ & $1154,3 \pm 224,9$ \\
\hline \multirow{2}{*}{ Sex } & Male & 30 & 21 & 9 \\
\cline { 2 - 5 } & Female & 14 & 22 & 12
\end{tabular}

Table 2 Relationship between retinopathy of prematurity and risk factors

\begin{tabular}{|c|c|c|c|}
\hline \multirow{2}{*}{ Risk factors } & \multicolumn{2}{|l|}{ Retinopathy of prematurity } & \multirow{2}{*}{$\mathrm{p}$} \\
\hline & Without, I and II stage (n) & Severe (n) & \\
\hline \multicolumn{4}{|c|}{ Gestational age (weeks) } \\
\hline$<30$ & 21 & 16 & \multirow{2}{*}{$<0.01$} \\
\hline$>30$ & 66 & 5 & \\
\hline \multicolumn{4}{|l|}{ Birth weight } \\
\hline$<1250 \mathrm{~g}$ & 13 & 13 & \multirow{2}{*}{$<0.01$} \\
\hline$>1250 \mathrm{~g}$ & 74 & 8 & \\
\hline \multicolumn{4}{|c|}{ Oxygen therapy } \\
\hline Yes & 79 & 21 & \multirow{2}{*}{$<0.001$} \\
\hline No & 8 & 0 & \\
\hline \multicolumn{4}{|c|}{ Respiratory distress syndrome } \\
\hline Yes & 40 & 16 & \multirow{2}{*}{0.013} \\
\hline No & 47 & 5 & \\
\hline \multicolumn{4}{|c|}{ Mechanical ventilation } \\
\hline Yes & 26 & 17 & \multirow{2}{*}{$<0.05$} \\
\hline No & 61 & 4 & \\
\hline \multicolumn{4}{|l|}{ Apnoea } \\
\hline Yes & 20 & 17 & \multirow{2}{*}{$<0.001$} \\
\hline No & 67 & 4 & \\
\hline \multicolumn{4}{|c|}{ Perinatal asphyxia } \\
\hline Yes & 9 & 9 & \multirow{2}{*}{$<0.001$} \\
\hline No & 78 & 12 & \\
\hline
\end{tabular}




\begin{tabular}{|c|c|c|c|c|}
\hline \multirow{3}{*}{ Variable } & \multicolumn{3}{|c|}{ Retinopathy of prematurity } & \multirow{3}{*}{$\mathrm{p}$} \\
\hline & Without & I i II stage & Severe & \\
\hline & $\mathrm{n}=44$ & $\mathrm{n}=43$ & $\mathrm{n}=21$ & \\
\hline Duration of $\mathrm{O}_{2}$ (days; $\overline{\mathrm{x}}$; range) & $6.4(0-15)$ & $21.9(11-44)$ & $44.5(27-73)$ & $<0.01$ \\
\hline Duration of MV (days; $\overline{\mathrm{x}}$; range) & $0.9(0-14)$ & $4.4(0-24)$ & $16.0(0-54)$ & $<0.05$ \\
\hline $\mathrm{FiO} 2(\% ; \bar{x} ;$ range $)$ & $23.3(21-28)$ & $29.4(24-38)$ & $43.0(31-51)$ & $<0.05$ \\
\hline Hyperoxia* n (\%) & $2(4.5)$ & $31(72)$ & $21(100)$ & $<0.01$ \\
\hline Hypoxia* n (\%) & $6(13.6)$ & $20(46.5)$ & $18(85.7)$ & $<0.01$ \\
\hline
\end{tabular}

$\mathrm{FiO}_{2}=$ Fraction of inspired oxygen; $\mathrm{MV}=$ Mechanical ventilation; ${ }^{*}$ Ten or more episodes.

100/108 (92.6\%) premature babies received oxygen therapy. During the treatment, $\mathrm{SaO}_{2}$ was restricted to $<92 \%$. Mechanical ventilation was used in $43 / 108$ babies $(39.81 \%)$ in the form of conventional mechanical ventilation and/or non-invasive positive pressure ventilation. The use of mechanical ventilation was statistically significantly more frequent in newborns who developed severe ROP $(\mathrm{p}<0.05)$ (Table 2).

In newborns who underwent oxygen therapy longer there was a statistically highly significant $(\mathrm{p}<0.001)$ greater incidence of severe $\mathrm{ROP}$. The average oxygen fraction value was analysed in the inspired air $\left(\mathrm{FiO}_{2}\right)$ in the first two weeks of life in all premature babies. We conclude that the incidence of severe $R O P$ is significantly greater $(p<0.05)$ in newborns who receive high concentrations of oxygen in the air they breathe. Also, it was established that frequent episodes of hyperoxia and hypoxia were highly significantly more frequent $(\mathrm{p}<0.001)$ in newborns with severe ROP (Table 3).

A multiple logistic regression model was designed for risk factors and it was established that there was an extremely strong connection between severe ROP and exposure to oxygen therapy, episodes of hyperoxia (OR 32.73; CI 4.14-255.37), episodes of hypoxia (OR 14.08; CI 3.81-51.94), oxygen therapy longer than 10 days (OR 15.54; CI 1.99120.79), frequent apnoea (OR 14.24; CI
4.30-47.19) and long term use of mechanical ventilation (OR 9.97; CI 3.06-32.51). In most of the risk factors examined there was a strong connection present between severe ROP and exposure to the risk factors: lower birth weight (OR 9.25; CI 3.20-26.69), lower gestational age (OR 10.06; CI 3.2930.76), respiratory distress syndrome (OR 3.76; CI 1.26-11.17) and perinatal asphyxia (OR 6.50; CI 2.15-19.64) (Table 4).

Table 4 Multiple logistic regression analysis of risk factors related to retinopathy of prematurity

\begin{tabular}{llll}
\hline Risk factors & OR & $95 \% \mathrm{CI}$ & $\mathrm{p}$ \\
\hline GA $<30$ weeks & 10.06 & $3.29-30.76$ & $<0.001$ \\
$\mathrm{BW}<1250 \mathrm{~g}$ & 9.25 & $3.20-26.69$ & $<0.001$ \\
Oxygen therapy* $^{*}$ & 15.54 & $1.99-120.79$ & $<0.001$ \\
Mechanical ventilation & 9.97 & $3.06-32.51$ & $<0.001$ \\
Hyperoxia $\dagger$ & 32.73 & $4.14-255.37$ & $<0.001$ \\
Hypoxia $\dagger$ & 14.08 & $3.81-51.94$ & $<0.001$ \\
RDS & 3.76 & $1.26-11.17$ & 0.013 \\
Apnoea & 14.24 & $4.30-47 . .19$ & $<0.001$ \\
Perinatal asphyxia & 6.50 & $2.15-19.64$ & $<0.001$ \\
\hline
\end{tabular}

$\mathrm{OR}=\mathrm{Odds}$ ratio; $\mathrm{CI}=$ confidence interval; $\mathrm{GA}=\mathrm{Gestational}$ age; *Ten or more days; $\dagger$ Ten or more episodes; RDS=respiratory distress syndrome.

\section{Discussion}

This study confirms that ROP still occurs in a significant number of premature babies. The use of oxygen and episodes of hyperoxia and 
hypoxia in premature babies with low birth weight are not the only or most important risk factors, but testing the effect of oxygen as an aetiological factor for the development of ROP is still important. ROP is a potentially serious condition which may lead to blindness, and in its treatment long-term monitoring is necessary due to the increased tendency to short-sightedness, strabismus and poor sight. For this reason all procedures that lead to a reduction in the development of severe ROP are important. Reduction of the incidence of ROP is achieved by education and changes to practice, so that controlled and limited $\mathrm{SaO}_{2}$ is used, measured by a pulse oximeter (10).

The incidence of severe ROP in our study over a two-year period was 21/108 (19.4\%) premature babies who met the criteria for screening for ROP. Developing countries, which include this country, show the presence of severe ROP with higher gestational age and greater birth weight, but the incidence varies greatly $(11,12)$. In our study, in the test group of newborns there was no severe ROP where the birth weight was $>1460$ $\mathrm{g}$ and gestation $>31.4$ weeks, but we had a smaller number of newborns under $1000 \mathrm{~g}$. We can explain this by the lower survival rate of babies with very low birth weight in our conditions. Similar results were also obtained in other developing countries (13), whilst in developed countries, severe ROP occurs in babies with very low birth weight and lower gestational age (14).

Low gestational age was a statistically highly significant factor $(\mathrm{p}<0.01)$ for occurrence of ROP in our study. The average age in the group with severe ROP was $28.8 \pm 1.5$ weeks. The incidence of ROP in newborns BW $<1250$ g was $20.3 \%$ (13/64), and with $\mathrm{BW}>1250 \mathrm{~g}$ the incidence was $12.5 \%$. All studies point out that a significant risk factor is premature birth, and the incidence of ROP increases with prematurity $(15,16)$.
In our study, in newborns who underwent oxygen therapy for a longer time, the incidence of severe ROP was statistically highly significantly more frequent $(\mathrm{p}<0.01)$. Long-term and repeated exposure to hyperoxia is linked to bronchopulmonary dysplasia and ROP in newborns with very low birth weight. Bizzarro et al. (17) showed a significant reduction in severe ROP after the introduction of specific target values of $\mathrm{SaO}_{2}$, education of staff on the potential risks due to exposure to oxygen, and the introduction of new technology using pulse oximeters. Alongside hyperoxia, the duration of use of oxygen therapy is also shown in many studies as a risk factor for development of ROP (18).

The optimum level of oxygen in treatment of premature babies is still being sought. Control of oxygenation is achieved by halting the use of high concentrations of oxygen. Lower concentrations significantly reduce the incidence of severe ROP, as shown in the study by Wrigh et al. (19). Due to the harmful effects of hyperoxia, the attitude has changed towards the use of supplementary oxygen in resuscitation of newborns. However, on the basis of the current knowledge, resuscitation is begun with room air, and additional oxygen is used if necessary (20). There is a large amount of evidence to show that resuscitation using room air reduces mortality and morbidity. In a recently published study, Rabi et al. (21), monitored the consequences of resuscitation using high concentrations of $100 \%$ oxygen, titrated, moderate concentrations (30-90\%) and room air, until saturation between $85 \%$ to $92 \%$ was achieved, for the occurrence of ROP. They concluded that the best results came from using titrated, moderate concentrations of oxygen.

$\mathrm{SaO}_{2}$ provides information about the adequacy of respiratory function, and can be assessed non-invasively using a pulse oximeter. In all premature babies in our study, who were on oxygen therapy, $\mathrm{SaO}_{2}$ was constantly 
monitored and was $<92 \%$. The importance is well-known of using a pulse oximeter in continuous assessment of $\mathrm{SaO}_{2}$, and in recent studies the importance of the technical improvement and sensitivity of the apparatus has been shown. Castillo et al. (10) recommend the maintenance of a constant level of saturation in premature babies $<1250 \mathrm{~g}$, in order to reduce hyperoxia, which would have a significant effect on reducing the incidence of severe ROP. The precision of measurement of $\mathrm{SaO}_{2}$ is insufficient in critically ill newborns and where there are high values of partial pressure oxygen in the blood.

In most studies the value of $\mathrm{SaO}_{2}$ in premature babies at $<32 \mathrm{GW}$ is between $89-94 \%$, in some it is $85-95 \%$. The use of controlled and limited $\mathrm{SaO}_{2}$ induced a reduction in the incidence of severe ROP (22), but there was an increase in mortality (23). Wallance et al. (24) tried to establish the incidence of severe ROP with the use of slightly reduced levels of oxygen saturation of $90-96 \%$ in the first few weeks of life. They noticed that there was a low statistical significance in the reduction of the incidence of severe ROP with the use of slightly lower oxygen saturation. In a recently published study, Waldemar et al. (25), observed in premature babies born at 24-27 GW the effect of the use of lower oxygen saturation $(85-89 \%)$, in comparison with higher levels $(91-95 \%)$ on the development of ROP. They did not notice a reduction in ROP with the use of lower oxygen saturation, but they did notice an increase in mortality. A restrictive approach to oxygen therapy requires prospective monitoring and an assessment of the benefits and harm, in major meta-analysis studies (26). Recently published studies recommend the use of supplementary oxygen in premature babies with gestation $\geq 32$ GW during the first few weeks of life, with lower oxygen saturation (85-93\%), whilst later than $\geq 32 \mathrm{GW}$ they should have higher oxygen saturation (94-99\%). This approach to treatment with oxygen is accompanied by a reduction in the risk of occurrence of severe forms of $\operatorname{ROP}(23,26)$.

In our study, we established that the incidence of severe ROP was significantly higher $(p<0.05)$ in babies who received higher concentrations of oxygen during the first two weeks of life (Table 3). Similar results to ours were presented in the study by Al-Essa et al. (27). Hauspurg et al. (28) found that high concentrations of oxygen and carbon dioxide in the blood and low $\mathrm{pH}$ were accompanied by an increased risk of development of ROP. Further research is needed to explain the connection between oxygenation, ventilation and acidosis.

The results of our study show that premature babies who received oxygen and who had frequent episodes of hyperoxia and hypoxia, had a severe form of ROP statistically highly significantly more often $(p<0.01)$. Exposure to hyperoxia, even for a short time after birth may lead to oxidative stress, which can result in apoptosis and the death of cells. Wangsa-Wirawan et al. (29) published that tissue damage was induced by both hyperoxia and hypoxia. A great deal is known about the short-term and long-term consequences of hyperoxia, but not so much about the effects of hypoxia. Experiments have shown that fluctuations in oxygen saturation in arterial blood, even within values considered to be normal, may increase the incidence of ROP, but also reduce it (30).

In our study, long duration of use of mechanical ventilation was significantly more frequent $(p<0.05)$ in the group of newborns with severe ROP (Table 3). Many authors point out that longer use of mechanical ventilation is associated with the development of severe forms of $\operatorname{ROP}(16,31,32)$. In a recently published study, Finer et al. (33) showed the lower incidence of ROP with restricted use of mechanical ventilation, and with the use of non-invasive positive pressure 
ventilation. In our study, the incidence of respiratory distress due to Hyaline Membrane Disease was greater $(\mathrm{p}<0.05)$ in newborns who developed severe ROP. In many studies it has been confirmed that RDS is linked to the development of $\operatorname{ROP}(16,34,35)$.

Using multiple logistic regression analysis, we obtained predictive factors with a strong connection to the occurrence of severe ROP, which are: exposure to long-term oxygen therapy (OR, 15.54; CI, 1.99-120.79), frequent episodes of hyperoxia (OR, 32.73; CI, 4.14-255.37), frequent apnoea (OR 14.24; CI 4.30-47.19) and longer use of mechanical ventilation (OR, 9.97; CI, 3.06-32.51). The duration of oxygen therapy and higher concentrations of oxygen are directly linked to the length of mechanical ventilation. Our results are in agreement with the results of Shah et al. (16). There is a slightly lesser connection with the development of severe ROP and lower birth weight, lower gestational age, RDS and perinatal asphyxia.

In a paper from 2014, Vento (36) showed, in 5000 newborns with very low birth weight and gestation $<28 \mathrm{GW}$, that premature babies who have $\mathrm{SaO}_{2}$ in the region of $91-95 \%$ up to $36 \mathrm{GW}$, have significantly lower mortality and fewer severe disabilities than newborns with low $\mathrm{SaO}_{2}$ (85-89\%). It is still not known whether $\mathrm{SaO}_{2}$ should be constant during this post-natal period, or if it should be reduced or even increased during infections, stagnation in growth, ROP and BPD. A prospective multi-centric protocol is being drawn up for neonatal oxygenation, which will provide guidelines for the use of the most reliable concentrations of oxygen (37).

The key guideline for a reduction in the incidence of ROP is avoiding hyperoxia early in the life of newborns $(35,38)$. Premature babies should be resuscitated with an oxygen mixture $(30-90 \%)$ so that saturation is gradually increased in the first 10 minutes of life (39). It is recommended to keep $\mathrm{SaO}_{2}$ val- ues constant over the first few weeks of life, but not below $90 \%$ (26). Further research is needed to establish a combination of various methods that may be used to reduce mortality, alongside a reduction in the incidence of severe ROP (40).

\section{Conclusion}

In our study, several risk factors were seen to cause the development of ROP: long-term oxygen therapy, hyperoxia, hypoxia, low gestational age and low birth weight. Also, the illnesses that develop in premature babies through mechanisms related to oxygen cause the development of severe ROP are: respiratory distress syndrome, frequent apnoea and perinatal asphyxia. It is necessary to work on the prevention of premature birth, and use oxygen therapy and mechanical ventilation sensibly, and in this way it is possible to reduce the incidence and severity of ROP.

Authors' contributions: Conception and design: DJ, MM; Acquisition, analysis and interpretation of data: DJ, MM; Drafting the article DJ, MM, VM; Revising it critically for important intellectual content: DJ, JPS, DN.

Conflict of Interest: The authors declare that they have no conflict of interest.

\section{References}

1. Jacobson RM, Feinstein AR. Oxygen as a cause of blindness in premature infants: Autopsy of a decade of errors in clinical epidemiologic research. J Clin Epidemiol. 1992;45(11):1265-87.

2. Coats DK and Reddy AK. Retinopathy of Prematurity. In: Wilson ME, et al. editors. Pediatric Ophthalmology. Berlin: Springer-Verlag; 2009. p 375-86.

3. Robertson AF. Reflections on errors in neonatology: The „hands-off“ years 1920 to 1950 . J Perinatol. 2003;23:48-55.

4. Flynn JT, Bancalari E, Snyder ES, Goldberg RN, Feuer W, Cassady J, et al. A Cohort study of transcutaneous oxygen tension and the incidence and 
severity of retinopathy of prematurity. Tm Am Ophth Soc. 1991;89:77-95.

5. Fleck BW and McIntosh N. Pathogenesis of retinopathy of prematurity and possible preventive strategies. Early Hum Dev. 2008;84(2):83-8.

6. Cavallaro G, Filippi L, Bagnoli P, La Marca G, Cristofori G, Raffaeli G, et al. The pathophysiology of retinopathy of prematurity: an update of previous and recent knowledge. Acta Ophthalmol. 2014;92(1):2-20.

7. Ikeda H, Kuriyama S. Risk factors for retinopathy of prematurity requiring photocoagulation. Jpn J Ophthalmol. 2004;48(1):68-71.

8. Mintz-Hittner HA, Kennedy KA, Chuang AZ for the BEAT-ROP Cooperative Group. Efficacy of IntravitrealBevacizumab for Stage 3+ Retinopathy of Prematurity. N Engl J Med. 2011;364(7):603-15.

9. International Committee for the classification of retinopathy of prematurity. The International classification of retinopathy of prematurity revisited. Arch Ophthalmol. 2005; 123(7):991-9.

10. Castillo A, Deulofeut R, Critz A and Sola A. Prevention of retinopathy of prematurity in preterm infants through changes in clinical practice and $\mathrm{SpO} 2$ technology. Acta Paediatr. 2011;100(2):188-92.

11. Karna P, Muttineni J, Angell L, and Karmaus W. Retinopathy of prematurity and risk factors: a prospective cohort study. BMC Pediatr. 2005;5(1):18.

12. Babaei H, Ansari MR, Alipour AA, Ahmadipour S, Safari-Faramani R and Vakili J. Incidence and Risk Factors for Retinopathy of Prematurity in Very Low Birth Weight Infants in Kermanshah, Iran. World Appl Sci J. 2012;18(5):600-4.

13. Ahmed AS, Muslima H, Anwar KS, Khan NZ, Chowdhury M, Saha SK, et al. Retinopathy of prematurity in Bangladeshi neonates. J Trop Pediatr. 2008;54(5): 333-9.

14. Lad EM, Hernandez-Boussard T, Morton JM, Moshfeghi DM. Incidence of Retinopathy of Prematurity in the United States: 1997 through 2005. Am J Ophthalmol. 2009;148:451-8.

15. Sapieha P, Joyal JS, Rivera JC, Kermorvant-Duchemin E, Sennlaub F, Hardy P, et al. Review series Retinopathy of prematurity: understanding ischemic retinal vasculopathies at an extreme of life. Pediatrics. 2010;120(9):3022-33.

16. Shah VA, Yeo CL, Ling YLF, Ho LY. Incidence, risk factors of retinopathy of prematurity among very low birth weight infants in Singapore. Ann Acad Med Singapore. 2005;34(2):169-78.
17. Bizzarro MJ, Li FY, Katz K, Shabanova V, Ehrenkranz R, Bhandari V. Temporal quantification of oxygen saturation ranges: an effort to reduce hyperoxia in the neonatal intensive care unit. J Perinatol. 2014;34(1):33-8.

18. Chen Y, Li XX, Gilbert C, Liang JH, Jiang YR, Zhao MW. Beijing ROP Survey Group. Risk factor for retinopathy of prematurity in six neonatal intensive care units in Beijing, China. Br J Ophthalmol. 2008;92:326-31.

19. Wrigh KW. A physiologic reduced oxygen protocol decreases the incidence of threshold. Trans Am Ophthalmol Soc. 2006;104:78-84.

20. Saugstad OD, Ramji S, Soll RF, Vento M. Resuscitation of Newborn Infants with $21 \%$ or $100 \%$ Oxygen: An Updated Systematic Review and Meta-Analysis. Neonatology. 2008;94:176-82.

21. Rabi Y, Singhal N, Nettel-Aguirre A. Room-air versus oxygen administration for resuscitation of preterm infants: the ROAR study. Pediatrics. 2011;128:374-81.

22. Chang M. Optimal oxygen saturation in premature infants. Korean J Pediatr. 2011;54(9):359-62.

23. Chen ML, Guo L, Smith LE, Dammann CE, Dammann O. High or low oxygen saturation and severe retinopathy of prematurity: a meta-analysis. Pediatrics. 2010;125(6):1483-92.

24. Wallance DK, Veness-Meehan KA, Miller WC. Incidence of Severe Retinopathy of Prematurity Before and After a Modest Reduction in Target Oxygen Saturation Levels. Manuscript, Author. J AAPOS. 2007;11(2):170-4.

25. Waldemar AC, Neil NF, Michele CW, Wade R, Marie GG, Abbot RL, et al. Target Ranges of Oxygen Saturation in Extremely Preterm Infants. SUPPORT Study Group of the Eunice Kennedy Shriver NICHD Neonatal Research Network. N Engl J Med. 2010;362(21):1959-69.

26. Saugstad OD, Aune D. Optimal Oxygenation of Extremely Low Birth Weight Infants: A MetaAnalysis and Systematic Review of the Oxygen Saturation Target Studies. Neonatology. 2014;105:5563.

27. Al-Essa M, Azad RV, Rashwan N. Threshold stage of retinopathy of prematurity: maternal and neonatal risk factors. Ann Saudi Med. 2000;20(2):12931.

28. Hauspurg AK, Allred EN, Vanderveen DK, Chen $\mathrm{M}$, Bednarek FJ, Cole C, et al. Blood gases and retinopathy of prematurity: the ELGAN Study. Neonatology. 2011;99(2):104-11. 
29. Wangsa-Wirawan ND, Linsenmeier RA. Retinal Oxygen: Fundamental and Clinical Aspects. Arch Ophthalmol. 2003;121:547-57.

30. Sola A, Golombek SG, Montes Bueno MT, Lemus-Varela L, Zuluaga C, Dominguez F, et al. Safe oxygen saturation targeting and monitoring in preterm infants: can we avoid hypoxia and hyperoxia. Acta Pediatr. 2014;103:1009-18.

31. Sarikabadayi YU, Aydemir O, Ozen ZT, Aydemir C, Tok L, Oguz SS, Erdeve O, et al. Screening for retinopathy of prematurity in a large tertiary neonatal intensive care unit in Turkey: frequency and risk factors. Ophthalmic Epidemiol. 2011:18(6):26974.

32. Kim TIm, Sohn J, Pi SYoung, Yoon YH. Postnatal risk factors of retinopathy of prematurity. Paediatr Perinat Epidemiol. 2004;18(2):130-4.

33. Finer NN, Carlo WA, Walsh MC, Rich W, Gantz MG, Laptook AR, et al. Early CPAP versus Surfactant in Extremely Preterm Infants. SUPPORT Study Group of the Eunice Kennedy Shriver NICHD Neonatal Research Network. N Engl J Med. 2010;362(21):1970-9.

34. Vinekar A, Dogra MR, Sangtam T, Narang A, Gupta A. Retinopathy of prematurity in Asian Indian babies weighing greater than 1250 grams at birth: Ten year data from a tertiary care center in a developing country. Indian J Ophthalmol. 2007;55(5):331-6.

35. Alpay A, Uğurbaş SH. Incidence and Risk Factors for Retinopathy of Prematurity in the West Black Sea Region, Turkey. Turk J Pediatr. 2012;54:113-8.

36. Vento M. Oxygen supplementation in the neonatal period: changing the paradigm. Neonatology. 2014;105(4):323-31.

37. Askie LM, Brocklehurst P, Darlow BA, Finer N, Schmidt B, Tarnow-Mordi W. NeOProM: Neonatal Oxygenation Prospective Meta-analysis Collaboration study protocol. BMC Pediatrics. 2011;11(1):6.

38. Fortes Filho JB, Valiatti FB, Eckert GU, Costa MC, Silveira RC, Procianoy RS. Is being small for gestational age a risk factor for retinopathy of prematurity? A study with 345 very low birth weight preterm infants. J Pediatr (Rio J). 2009;85(1):4854.

39. Rook D, Schierbeek H, van der Eijk AC, Longini M, Buonocore G, Vento M, et al. Resuscitation of very preterm infants with $30 \%$ vs. $65 \%$ oxygen at birth: study protocol for a randomized controlled trial. Trials. 2012;13:65.

40. Hartnett ME, Lane RH. Effects of oxygen on the development and severity of retinopathy of prematurity. J AAPOS. 2013;17(3):229-34. 